\title{
Nano for agriculture, not the opposite
}

\author{
Although the idea of using nanomaterials for agriculture is promising, we must consider in detail how \\ nanotechnology can provide advantages over standard tools.
}

\begin{abstract}
A griculture is an environmentally costly technology. A growing population and adverse climate conditions increases the need to use fertilizers and pesticides. These however, tend to be very inefficient, resulting in potentially toxic substances being released into the environment in large amounts. Fertilizers and pesticides based on nanomaterials could provide an important solution to the problem. The principle is simple: because nanomaterials are so small, they can release the desired substance locally, thus reducing waste.

The interest in nano-enabled agriculture is growing rapidly. A fast analysis on SCOPUS shows that the number of publications in the field has risen almost tenfold in the last 10 years. Beyond reporting results based on performance, researchers are focusing more and more on understanding the mechanisms of interaction between the nanomaterials and
\end{abstract}

crops, which provides interesting scientific insight and is essential for the successful development of the field. Indeed, providing insight into such interaction mechanisms has now become an essential ingredient for the editorial team at Nature Nanotechnology to consider a manuscript for publication and seek the advice of reviewers.

Although advances in science and applications are crucial for the development of nanotechnology, it is also essential to assess whether and how exactly the use of nanomaterials is going to provide advantages with respect to other approaches. The Analysis by Leanne Gilbertson and colleagues is a clear example of such type of study. Starting from the assumption that nanomaterials should provide advantages in terms of environmental costs, the team used a life-cycle assessment approach applied to previously published data on the use of nanofertilizers to estimate whether the total energy, including that needed for nanomaterial production besides their applications, and compared it with that of non-nano fertilizers.

Analyses such as that by Gilbertson and colleagues are especially valuable to provide direction to the community. In the specific case, the study shows that adding nanofertilizers to the soil is not overall an environmentally friendly strategy, while designing nanoparticles to be used in seed coating or foliar sprays could be. Such studies are also essential to rebut one of the main criticisms often posed to nanotechnology, namely, that experts in nanomaterials often need to find an application to justify their research. We can now be more confident that if properly designed, nanoparticles can really provide solutions to challenges in agriculture.

Published online: 8 September 2020 https://doi.org/10.1038/s41565-020-0766-6

\section{Enter plastic}

Two studies on sub-micrometre plastic particles provide evidence of plastic accumulation in terrestrial plants.

$\mathrm{P}$ lastic waste is attracting growing research attention, especially in the environmental science community. It is also a topic that can trigger the interest of the general public because of its high visual impact. Most research efforts so far have focused on monitoring the fate of plastic microparticles, and to a certain extent nanoparticles, in water, and their interaction with aquatic organisms, in the attempt to estimate how much plastic such organisms uptake and ultimately how toxic the plastic can be.

Comparatively, less attention has been given to the uptake of plastic particles by terrestrial plants. This is however becoming quite relevant for the potential negative effect that plastic can have on the environment generally, and more specifically on agriculture. The Letter by Xiao-Dong Sun and co-workers now published in Nature Nanotechnology, and the Article by Lianzhen Li and co-workers now published in Nature Sustainability are significant advances in our knowledge because they report experimental evidence of plastic particle uptake by a number of terrestrial plants.

The work by Sun et al. focuses on how the charge of plastic nanoparticles around $200 \mathrm{~nm}$ in size affects the uptake by the standard model species Arabidopsis thaliana. Artificially synthesized plastic nanoparticles were added to the soil. Positively charged particles were then seen accumulating in the roots and even affecting root growth, while negative particles were observed also in the plant vasculature

Li et al. focused on particles ranging from $200 \mathrm{~nm}$ to a few micrometres and they studied the uptake in wheat and lettuce.
Through high-resolution scanning electron microscopy, it was possible to establish that plastic particles of different sizes enter the plants by the so-called crack-entry mechanism, through openings in the roots that are also known to be entry points for bacteria in plants developing infections.

The ultimate goal of research on plastic particles is to understand how toxic they can be for organisms in the environment and, to be fair, neither result answers that question yet. But knowing the details of how much and through which mechanism the particles accumulate in plants is a crucial first step and provides an important starting point for studies of toxicity.

Published online: 8 September 2020 https://doi.org/10.1038/s41565-020-0767-5 